\title{
Efeitos de Diferentes Condições de Umidade do Solo e PROFUndIDAdES DE GERMINAÇÃo DE Brachiaria plantaginea E Digitaria spp. SOBRE A EFICÁCIA DO HERBICIDA TEBUTHIURON ${ }^{1}$
}

\author{
Effect of Soil Humidity Conditions and Germination Depths of Brachiaria plantaginea and \\ Digitaria spp. on Tebuthiuron Efficacy
}
NEGRISOLI, E. ${ }^{2}$, CARBONARI, C.A. ${ }^{3}$, CORREA, M.R. ${ }^{4}$, PERIM, L. ${ }^{5}$, VELINI, E.D. ${ }^{6}$, TOLEDO, R.E.B. ${ }^{7}$, VICTORIA FILHO, R. ${ }^{8}$ e ROSSI, C.V.S. ${ }^{9}$

\begin{abstract}
RESUMO - Com o objetivo de avaliar a eficácia do herbicida tebuthiuron no controle de Brachiaria plantaginea e Digitaria spp. em função da profundidade de germinação, condição de umidade do solo e período de seca, realizou-se um experimento em casa de vegetação. Os tratamentos foram constituidos de cinco profundidades de germinação das plantas daninhas $(1,2,3,4$ e $5 \mathrm{~cm})$ e aplicação do produto sob quatro condições de umidade do solo, sendo o tratamento 1: $15 \mathrm{~mm}$ de chuva, seguido de aplicação; tratamento 2: $15 \mathrm{~mm}$ de chuva, seguido de aplicação e $15 \mathrm{~mm}$ de chuva sete dias após a aplicação (DAA); tratamento 3: $15 \mathrm{~mm}$ de chuva, seguido de aplicação e $15 \mathrm{~mm}$ de chuva aos 14 DAA; tratamento 4: aplicação do produto em solo seco e $15 \mathrm{~mm}$ de chuva aos 14 DAA; e uma testemunha sem aplicação do herbicida, dispostos em um esquema fatorial $5 \times 5$, com quatro repetições. As sementes das plantas daninhas foram semeadas em vasos nas diferentes profundidades de germinação. Foram realizadas avaliações visuais de controle aos 14, 28 e 42 dias após a aplicação (DAA); ao final do estudo, foi avaliada a biomassa seca da parte aérea das plantas. O herbicida promoveu controle efetivo das plantas de $B$. plantaginea em todas as profundidades testadas e condições de aplicação, permitindo falha significativa no controle para as plantas que germinaram a $5 \mathrm{~cm}$ de profundidade quando o produto foi aplicado em solo úmido e sem ocorrência de chuvas. Para Digitaria sp., verificaram-se excelentes niveis de controle das plantas inicialmente e falhas no controle para aplicação em solo úmido, seguido da ocorrência de chuvas aos 14 DAA nas profundidades de 2 e $3 \mathrm{~cm}$, o que demonstra uma possivel lixiviação desse herbicida para camadas abaixo de $3 \mathrm{~cm}$ no solo.
\end{abstract}

Palavras-chave: planta daninha, lixiviação, mobilidade.

\begin{abstract}
This work aimed to carry out an experiment under greenhouse conditions to evaluate the effect of humidity conditions and germination depth of Brachiaria plantaginea and Digitaria spp. on the efficacy of tebuthiuron. The treatments were constituted by five germination depths of the weeds and different herbicide application conditions $(1 ; 2 ; 3 ; 4$ and $5 \mathrm{~cm})$ and four humidity conditions. The following treatments were applied: treatment 1: $15 \mathrm{~mm}$ of rainfall, followed by herbicide application; treatment 2: $15 \mathrm{~mm}$ of rainfall, followed by herbicide application and $15 \mathrm{~mm}$ of rainfall after 7 days; treatment 3: $15 \mathrm{~mm}$ of rainfall, followed by herbicide application and $15 \mathrm{~mm}$ of rainfall after 14 days; and treatment 4: herbicide application on dry soil and $15 \mathrm{~mm}$ of rainfall after 14 days, and a control without application. The treatments were arranged in a $5 \times 5$ factorial scheme, with four repetitions. The weeds were seeded in vases at the different depths. Tebuthiuron
\end{abstract}

1 Recebido para publicação em 15.7.2009 e aprovado em 30.3.2011.

2 Engo-Agro-., Dr., Techfield - Consultoria Agrícola/Pos-Doutorando Nupam/FCA-Unesp, Botucatu-SP, Prof. Dr., Faculdade de Agronomia Eduvale/Avaré/SP, <eduardo.negrisoli@gmail.com>; ${ }^{3}$ Professor, Dr., FCA-Unesp, Botucatu-SP, <carbonari@fca.unesp.br>; ${ }^{4}$ Engo-Agr ${ }^{\circ}$., Dr., Techfield - Consultoria Agrícola; Pos-Doutorando Nupam/FCA-Unesp, Botucatu-SP, <marcelorcorrea@uol.com.br>; ${ }^{5}$ Doutorando Nupam/FCA-Unesp, Botucatu-SP, <lperim@fca.unesp.br>; ${ }^{6}$ Professor, Dr., Adjunto, Dep. de Agricultura, FCA/Unesp, Fazenda Experimental Lageado, Caixa Postal 237, 18603-970 Botucatu-SP, <velini@fca.unesp.br>; ${ }^{7}$ Engo-Agr ${ }^{\circ}$., Dr., Rua Dona Santina, 967, 13405-367 Piracicaba-SP, <roberto.estevao@uol.com.br>; ${ }^{8}$ Prof. Titular do Dep. de Produção Vegetal da ESALQ/USP, <rvictori@esalq.usp.br>; ${ }^{9}$ Engo-o-Agr ${ }^{0}$., Dr., Nupam/FCA-Unesp, Botucatu-SP, <cavsragro@msn.com>.

Planta Daninha, Viçosa-MG, v. 29, p. 1061-1068, 2011. Número Especial 
promoted an effective control of the plants of B. plantaginea at all depths tested and application conditions, with a significant flaw in the control of the plants that germinated at $5 \mathrm{~cm}$ depth where the herbicide was applied to wet soil and without rainfall. For Digitaria spp., excellent plant control levels were initially observed, with flaws in the control for wet soil application, followed by rainfall occurrence at 14 DAA at depths of 2 and $3 \mathrm{~cm}$, indicating possible leaching of this herbicide for layers below $3 \mathrm{~cm}$ in the soil.

Keywords: weed, leaching, mobility.

\section{INTRODUÇÃO}

O banco de sementes de um solo pode ser considerado como a reserva de sementes e propágulos vegetativos tanto em profundidade quanto em sua superfície, constituindo a origem do ciclo de vida das espécies vegetais (Roberts, 1981; Fernandez-Quintanilla et al., 1991; Gazziero et al., 2001); ele não deve ser considerado um simples estoque de diásporos, pois apresenta dinâmica própria, a qual varia com a espécie, as condições da semente, a ocorrência de predadores e os fatores ambientais (Carmona \& Murdoch, 1995).

Dos fatores que determinam a passagem dos estádios fenológicos de semente para plântulas, a profundidade da semente no perfil do solo é um dos mais importantes e, sem dúvida, sua avaliação é essencial para o desenvolvimento de modelos de crescimento populacional da espécie e, com fins práticos, para avaliar a eficácia de métodos mecânicos, químicos, isolados ou associados, no controle das plantas daninhas (Canossa et al., 2007).

O uso de herbicidas também pode influenciar as espécies que compõem o banco de sementes, podendo aumentá-lo ou diminuí-lo, dependendo dos produtos utilizados (Ball, 1992). $\mathrm{O}$ número do banco de sementes de um solo cultivado continuamente com milho foi reduzido em aproximadamente $70 \%$ após três anos com aplicação de atrazina e cultivo nas entrelinhas. Quando se substituiu o herbicida por cultivos, o número de sementes aumentou cerca de 25 vezes mais em comparação com as parcelas com herbicida (Schweizer \& Zimdahl, 1984).

Entre os fatores bióticos, as plantas daninhas são um dos principais componentes do agroecossistema da cana-de-açúcar que interferem no desenvolvimento e na produtividade dessa cultura. A duração do tempo da competição determina prejuízos no crescimento e no desenvolvimento e, consequentemente, na produção das culturas (Zanine \& Santos, 2004). Esses autores relatam ainda que o grau de competição interespecífica depende de fatores relacionados à comunidade infestante (composição específica, população e distribuição) e da própria cultura (espécie ou cultivar, espaçamento entre linhas e população de semeadura).

O comportamento de um herbicida no solo é influenciado por processos de retenção, transformação e transporte do produto. A mobilidade do herbicida no solo, que influencia diretamente o seu desempenho no controle de plantas daninhas e a sua dissipação no ambiente, pode ser avaliada por diferentes métodos (Negrisoli et al., 2007; Silva et al., 2007). Entretanto, métodos que melhor se aproximem das condições de campo e que apresentem boa reprodutibilidade são os mais adequados para esse tipo de estudo (Firmino et al., 2008).

Segundo Javaroni et al. (1999), a biodisponibilidade de herbicidas aplicados ao solo é essencialmente governada pelo equilíbrio dinâmico existente entre os processos de adsorção dos compostos presentes nos coloides do solo e sua solubilidade na fase solo-água. Como consequência, uma considerável influência desses parâmetros sobre a ação seletiva desses herbicidas deve ser esperada.

Em diversas situações podem ser observadas as falhas no controle de diversas plantas daninhas em áreas tratadas com herbicidas residuais. Essas falhas podem estar relacionadas ao posicionamento do produto no perfil do solo, em função da ocorrência de chuvas. No entanto, após a ocorrência de chuvas, bons niveis de controle foram observados, independentemente da profundidade de germinação (Negrisoli et al., 2007, 2009). 
Nesse contexto, este trabalho teve como objetivo avaliar a eficácia do herbicida tebuthiuron no controle de Brachiaria plantaginea e Digitaria spp. em função da profundidade de germinação, da condição de umidade do solo e do período de seca.

\section{MATERIAL E MÉTODOS}

Foi conduzido um experimento em casa de vegetação no Núcleo de Pesquisas Avançadas em Matologia - NUPAM, da Faculdade de Ciências Agronômicas da UNESP, campus de Botucatu-SP. Os experimentos foram realizados em vasos plásticos com capacidade para $2 \mathrm{~kg}$ de solo e dimensões de $15 \times 15 \mathrm{~cm}$ $\left(0,0225 \mathrm{~m}^{2}\right)$ e $15 \mathrm{~cm}$ de profundidade. Os vasos foram preenchidos totalmente com solo do tipo Latossolo Vermelho distrófico (LVd) de textura média, adubado mediante análise prévia de suas características. As características granulométricas do solo eram: $20 \%$ de argila, $4 \%$ de silte e $76 \%$ de areia.

A dose do herbicida tebuthiuron (Lava 800 WG) aplicada foi de 1,2 kg i.a. ha-1 (dose segundo Rodrigues; Almeida, 2011). Nos tratamentos, foram avaliados os efeitos de cinco profundidades de germinação das plantas daninhas $(1,2,3,4$ e $5 \mathrm{~cm})$ e aplicação do produto sob quatro condições de umidade do solo, sendo o tratamento 1: $15 \mathrm{~mm}$ de chuva, seguido de aplicação; tratamento 2: $15 \mathrm{~mm}$ de chuva, seguido de aplicação e $15 \mathrm{~mm}$ de chuva sete dias após a aplicação (DAA); tratamento 3: $15 \mathrm{~mm}$ de chuva, seguido de aplicação e $15 \mathrm{~mm}$ de chuva aos 14 DAA; tratamento 4: aplicação do produto em solo seco e $15 \mathrm{~mm}$ de chuva aos 14 DAA; e uma testemunha sem aplicação do herbicida, dispostos em um esquema fatorial $5 \times 5$, com quatro repetições.

As plantas daninhas utilizadas foram Brachiaria plantaginea e Digitaria spp., sendo semeadas nas diferentes profundidades em quantidades suficientes para uma germinação mínima de 25 plantas por vaso, determinadas por teste de germinação. As sementes foram posicionadas nas diferentes profundidades e, posteriormente, os vasos foram preenchidos com solo.

A pulverização do herbicida e a simulação de chuva foram realizadas através de um equipamento instalado em laboratório do NUPAM, o qual é constituído de uma estrutura metálica com $3 \mathrm{~m}$ de altura por $2 \mathrm{~m}$ de largura, que permite acoplamento de um "carrinho" suspenso a 2,5 m de altura. A esse carrinho encontram-se acopladas duas barras de pulverização, uma responsável pelo sistema de simulação de chuva e a outra pelo sistema de pulverização de defensivos agrícolas, as quais se deslocam por uma área útil de $6 \mathrm{~m}^{2}$ no sentido do comprimento do equipamento. O tracionamento de ambas as barras é realizado através de correntes e engrenagens, com auxílio de um motor elétrico, cujo ajuste é dado por um modulador de frequência, permitindo a obtenção de velocidade constante previamente determinada. O sistema de pulverização tem funcionamento independente do sistema de simulação de chuva, apesar de ambos compartilharem o mesmo equipamento e funções de controle.

A simulação da chuva foi feita utilizandose uma bomba hidráulica de pressão constante e acionamento automático, a qual bombeia água armazenada de um reservatório, com capacidade para $1.000 \mathrm{~L}$, até a barra e pontas de pulverização responsáveis pela formação de gotas de chuva. Essa barra, situada a 1,45 m de altura em relação à superfície das unidades experimentais, é constituída por três bicos de pulverização TK-SS-20 de alta vazão, espaçados de $0,5 \mathrm{~m}$ e posicionados de forma a propiciar maior uniformidade de precipitação na área aplicada. Esse sistema foi operado com velocidade de deslocamento de $0,187 \mathrm{~km} \mathrm{~h}^{-1}$, o que correspondeu a 2,5 Hertz no modulador de frequência e pressão de trabalho de 0,81 $\mathrm{kgf} \mathrm{s}^{-2}$. Essas especificações proporcionaram a produção de gotas artificiais de chuva com diâmetro mediano volumétrico (DMV) de 1.140 micras, conforme informações do fabricante da ponta de pulverização (Spraying Systems Co.), e cada lâmina aplicada correspondeu a aproximadamente $2,5 \mathrm{~mm}$ de chuva simulada.

A barra de pulverização era constituída por quatro pontas de pulverização XR 11002 VS, espaçadas entre si de $0,5 \mathrm{~m}$ e posicionadas a $0,5 \mathrm{~m}$ de altura em relação à superfície das unidades experimentais. Para as pulverizações do herbicida, o sistema foi operado com velocidade de deslocamento de $3,6 \mathrm{~km} \mathrm{~h}^{-1}$, o que 
correspondeu a 45,0 Hertz no modulador de frequência, com consumo de calda correspondente a $200 \mathrm{~L} \mathrm{ha}^{-1}$. O equipamento foi operado sob pressão constante de 1,5 bar, pressurizado por ar comprimido. A manutenção da umidade dos vasos após simulação das chuvas foi realizada através de canos de PVC perfurados e posicionados no centro dos vasos, o que proporcionou o umedecimento subsuperficial dos vasos.

Foram realizadas avaliações aos 14, 28 e 42 dias após a aplicação (DAA), por meio de uma escala visual e percentual de notas, variando de zero a 100. Nesse caso, zero consistia em nenhum controle e 100 era a porcentagem de controle total das espécies daninhas. No final do estudo, foi determinada a biomassa seca da parte aérea destas. As avaliações foram realizadas até o desaparecimento ou a estabilização dos sintomas de fitointoxicação (SBCPD, 1995). Os resultados foram submetidos à análise de variância pelo teste $\mathrm{F}$ ( $5 \%$ de probabilidade), e as médias, comparadas pelo teste de Tukey ( $5 \%$ de probabilidade).

\section{RESULTADOS E DISCUSSÃO}

Verifica-se na Tabela 1 , aos 14 DAA, um bom comportamento no controle de $B$. plantaginea pelos tratamentos testados. Analisando o controle proporcionado pelos diferentes tratamentos, notam-se resultados significativamente inferiores para as sementes germinadas a partir de $4 \mathrm{~cm}$. Com exceção do tratamento em que o produto foi aplicado em solo úmido e precipitação posterior após sete dias, os demais tratamentos foram estatisticamente inferiores para as sementes germinadas a $5 \mathrm{~cm}$ de profundidade.

Nas avaliações ocorridas aos 28 DAA (Tabela 2), pode-se observar que, quando se compara o efeito dos tratamentos nas diferentes profundidades, o tratamento com aplicação em solo úmido e sem precipitação posterior foi o menos eficaz no controle de $B$. plantaginea em todas as profundidades. Nesse caso, nas profundidades de 2 e $5 \mathrm{~cm}$, as médias observadas foram estatisticamente inferiores.

Nos demais tratamentos, nota-se que a segunda precipitação, posterior à aplicação, independentemente do intervalo de ocorrência, foi fundamental para o incremento de eficácia do produto. Dessa forma, as médias observadas variaram de 95,5 a 100\%.

Comparando o comportamento individual dos tratamentos em relação à profundidade de germinação das plantas, com exceção do tratamento sem a ocorrência de chuva posterior à aplicação do herbicida, todos os demais não apresentaram diferença significativa em relação à profundidade. Assim, neste tratamento, a eficácia do produto sobre as sementes germinadas a $5 \mathrm{~cm}$ de profundidade foi estatisticamente inferior.

Tabela 1 - Controle das plantas de Brachiaria plantaginea nas diferentes profundidades de germinação pelo herbicida tebuthiuron, aos 14 DAA. Botucatu-SP - 2006/07

\begin{tabular}{|c|c|c|c|c|c|}
\hline \multirow{2}{*}{ Tratamento } & \multicolumn{5}{|c|}{ Profundidade $(\mathrm{cm})$} \\
\hline & 1 & 2 & 3 & 4 & 5 \\
\hline 1. Chuva de $15 \mathrm{~mm}$ e aplicação & $83,8 \mathrm{Aa}$ & $86,0 \mathrm{Aa}$ & $93,8 \mathrm{Aa}$ & $91,8 \mathrm{Aa}$ & $78,8 \mathrm{ABab}$ \\
\hline 2. Chuva (15 mm) - aplicação - chuva (15 mm) 7 DAA & $98,5 \mathrm{Aa}$ & $94,0 \mathrm{Aa}$ & $98,5 \mathrm{Aa}$ & $96,3 \mathrm{Aa}$ & $90,5 \mathrm{Aa}$ \\
\hline 3. Chuva (15 mm) - aplicação - chuva $(15 \mathrm{~mm}) 14$ DAA & $94,0 \mathrm{Aa}$ & $93,5 \mathrm{Aa}$ & $92,3 \mathrm{Aa}$ & $75,0 \mathrm{Bb}$ & $86,5 \mathrm{ABab}$ \\
\hline 4. Aplicação solo seco - chuva $(15 \mathrm{~mm}) 14 \mathrm{DAA}$ & $95,0 \mathrm{Aa}$ & $95,0 \mathrm{Aa}$ & $96,0 \mathrm{Aa}$ & $85,8 \mathrm{ABab}$ & $73,0 \mathrm{Bb}$ \\
\hline $\mathrm{F}$ (profundidade) & \multicolumn{5}{|c|}{$3,02 *$} \\
\hline $\mathrm{F}$ (umidade) & \multicolumn{5}{|c|}{$5,62 * *$} \\
\hline $\mathrm{F}$ (prof. $\mathrm{x}$ umidade) & \multicolumn{5}{|c|}{$3,42 * *$} \\
\hline CV (\%) & \multicolumn{5}{|c|}{12,96} \\
\hline DMS & \multicolumn{5}{|c|}{16,20} \\
\hline
\end{tabular}

Médias seguidas de mesma letra, maiúscula na coluna e minúscula na linha, não diferem estatisticamente entre si pelo teste $\mathrm{t}(\mathrm{P}<0,05)$.

${ }^{*}$ significativo a $5 \%$ de probabilidade; $* *$ significativo a $1 \%$ de probabilidade; ${ }^{\text {ns }}$ não significativo. 
Analisando os dados contidos na Tabela 3 (avaliações ocorridas aos 42 DAA), nota-se um mesmo comportamento dos tratamentos em relação às avaliações passadas. Novamente, observa-se que a ocorrência de precipitação posterior à pulverização foi imprescindivel para a maximização do potencial de controle do herbicida. Negrisoli et al. (2007) também observaram que a aplicação de tebuthiuron sobre o solo, após a ocorrência de chuva de $20 \mathrm{~mm}$ posteriormente à aplicação, resultou em excelente controle $(100 \%$ aos 21 dias após a aplicação) de $B$. plantaginea.

Dessa forma, o tratamento em que houve ausência de chuva posterior à aplicação foi significativamente menos eficaz que os demais sobre as sementes germinadas a $1 \mathrm{e}$ $5 \mathrm{~cm}$ de profundidade. Analisando esse tratamento isoladamente, novamente sua eficácia foi reduzida para as sementes germinadas na profundidade máxima deste estudo.

De maneira geral, observa-se que os resultados de massa seca (g) caracterizam os resultados observados de controle nos diferentes períodos de avaliação, destacando-se um bom acúmulo de biomassa de plantas nas testemunhas para todas as profundidades avaliadas e épocas de avaliação, o que pode ser comprovado na Tabela 7.

Em se tratando da espécie Digitaria spp., em avaliações ocorridas aos 14 DAA (Tabela 4), não foi observada diferença significativa para o comportamento dos diferentes tratamentos em nenhuma das profundidades testadas. Entretanto, ao contrário do observado para
B. plantaginea, melhores resultados foram encontrados para as sementes germinadas em maiores profundidades. Resultados significativamente inferiores foram observados, em todos os tratamentos, para sementes germinadas até $2 \mathrm{~cm}$ de profundidade. Dias et al. (2005), avaliando o controle da infestação natural de Digitaria nuda, observaram que aos 15 DAA o herbicida tebuthiuron proporcionou nivel de controle de 99\%, porém aos 60 DAA houve drástica redução no nivel de controle, chegando a $33 \%$.

Nas avaliações ocorridas aos 28 DAA (Tabela 5), analisando o comportamento dos tratamentos em relação à profundidade, com exceção da profundidade de $2 \mathrm{~cm}$, todas as demais profundidades de germinação não foram afetadas. Nesse caso, quando ocorreu chuva sete dias depois da aplicação do herbicida em solo úmido ou quando o produto permaneceu em solo seco por 14 dias, os resultados foram superiores, com médias de 99,5 e $100 \%$, respectivamente.

Analisando os tratamentos isoladamente, nos quais houve ausência de chuva posterior à pulverização, resultados médios significativamente inferiores foram encontrados nas profundidades menores: 98 e $91 \%$ para 1 e $2 \mathrm{~cm}$, respectivamente. A ocorrência de precipitação aos 14 DAA, quando aplicado em solo úmido, comprometeu significativamente a eficácia deste tratamento na profundidade de germinação de $2 \mathrm{~cm}$ (95\% de controle). Para os demais tratamentos, não foi observada diferença entre as profundidades.

Tabela 2 - Controle das plantas de Brachiaria plantaginea nas diferentes profundidades de germinação pelo herbicida tebuthiuron, aos 28 DAA. Botucatu-SP - 2006/07

\begin{tabular}{|c|c|c|c|c|c|}
\hline \multirow{2}{*}{ Tratamento } & \multicolumn{5}{|c|}{ Profundidade $(\mathrm{cm})$} \\
\hline & 1 & 2 & 3 & 4 & 5 \\
\hline 1. Chuva de $15 \mathrm{~mm}$ e aplicação & $88,3 \mathrm{Aab}$ & $84,3 \mathrm{Bab}$ & $91,5 \mathrm{Aa}$ & $92,3 \mathrm{Aa}$ & $73,8 \mathrm{Bb}$ \\
\hline 2. Chuva (15 mm) - aplicação - chuva $(15 \mathrm{~mm}) 7 \mathrm{DAA}$ & $98,8 \mathrm{Aa}$ & $95,8 \mathrm{Aa}$ & $99,0 \mathrm{Aa}$ & $99,8 \mathrm{Aa}$ & $95,5 \mathrm{Aa}$ \\
\hline 3. Chuva $(15 \mathrm{~mm})$ - aplicação - chuva $(15 \mathrm{~mm}) 14$ DAA & $99,5 \mathrm{Aa}$ & $100,0 \mathrm{Aa}$ & $97,5 \mathrm{Aa}$ & $96,3 \mathrm{Aa}$ & $97,3 \mathrm{Aa}$ \\
\hline 4. Aplicação solo seco - chuva $(15 \mathrm{~mm}) 14 \mathrm{DAA}$ & $99,3 \mathrm{Aa}$ & $100,0 \mathrm{Aa}$ & $98,3 \mathrm{Aa}$ & $90,5 \mathrm{Aa}$ & $92,0 \mathrm{Aa}$ \\
\hline $\mathrm{F}$ (profundidade) & \multicolumn{5}{|c|}{$1,63^{\mathrm{ns}}$} \\
\hline F (umidade) & \multicolumn{5}{|c|}{$17,13^{* *}$} \\
\hline F (prof. x umidade) & \multicolumn{5}{|c|}{$2,16^{*}$} \\
\hline $\mathrm{CV}(\%)$ & \multicolumn{5}{|c|}{8,90} \\
\hline DMS & \multicolumn{5}{|c|}{11,76} \\
\hline
\end{tabular}

Médias seguidas de mesma letra, maiúscula na coluna e minúscula na linha, não diferem estatisticamente entre si pelo teste $\mathrm{t}(\mathrm{P}<0,05)$ ${ }^{*}$ significativo a $5 \%$ de probabilidade; ${ }^{*}$ significativo a $1 \%$ de probabilidade; ${ }^{\text {ns }}$ não significativo. 
Tabela 3 - Controle das plantas de Brachiaria plantaginea nas diferentes profundidades de germinação pelo herbicida tebuthiuron, aos 42 DAA. Botucatu-SP - 2006/07

\begin{tabular}{|c|c|c|c|c|c|}
\hline \multirow{2}{*}{ Tratamento } & \multicolumn{5}{|c|}{ Profundidade $(\mathrm{cm})$} \\
\hline & 1 & 2 & 3 & 4 & 5 \\
\hline 1. Chuva de $15 \mathrm{~mm}$ e aplicação & $93,0 \mathrm{Ba}$ & $95,3 \mathrm{Aa}$ & $93,5 \mathrm{Aa}$ & $96,0 \mathrm{Aa}$ & $85,5 \mathrm{Bb}$ \\
\hline 2. Chuva (15 mm) - aplicação - chuva (15 mm) 7 DAA & $99,8 \mathrm{Aa}$ & $97,0 \mathrm{Aa}$ & $100,0 \mathrm{Aa}$ & $100,0 \mathrm{Aa}$ & $97,5 \mathrm{Aa}$ \\
\hline 3. Chuva (15 mm) - aplicação - chuva (15 mm) 14 DAA & $100,0 \mathrm{Aa}$ & $100,0 \mathrm{Aa}$ & $98,0 \mathrm{Aa}$ & $97,8 \mathrm{Aa}$ & $96,3 \mathrm{Aa}$ \\
\hline 4. Aplicação solo seco - chuva (15 mm) 14 DAA & $100,0 \mathrm{Aa}$ & $100,0 \mathrm{Aa}$ & $98,5 \mathrm{Aa}$ & $97,5 \mathrm{Aa}$ & $95,3 \mathrm{Aa}$ \\
\hline $\mathrm{F}$ (profundidade) & \multicolumn{5}{|c|}{$1,50^{\mathrm{ns}}$} \\
\hline F (umidade) & \multicolumn{5}{|c|}{$19,01 * *$} \\
\hline $\mathrm{F}$ (prof. $\mathrm{x}$ umidade) & \multicolumn{5}{|c|}{$2,39^{*}$} \\
\hline CV (\%) & \multicolumn{5}{|c|}{4,69} \\
\hline DMS & \multicolumn{5}{|c|}{6,4} \\
\hline
\end{tabular}

Médias seguidas de mesma letra, maiúscula na coluna e minúscula na linha, não diferem estatisticamente entre si pelo teste $\mathrm{t}(\mathrm{P}<0,05)$.

${ }^{*}$ significativo a $5 \%$ de probabilidade; $* *$ significativo a $1 \%$ de probabilidade; ${ }^{n s}$ não significativo.

Tabela 4 - Controle das plantas de Digitaria spp. nas diferentes profundidades de germinação pelo herbicida tebuthiuron, aos 14 DAA. Botucatu-SP - 2006/07

\begin{tabular}{|c|c|c|c|c|c|}
\hline \multirow{2}{*}{ Tratamento } & \multicolumn{5}{|c|}{ Profundidade $(\mathrm{cm})$} \\
\hline & 1 & 2 & 3 & 4 & 5 \\
\hline 1. Chuva de $15 \mathrm{~mm}$ e aplicação & $99,8 \mathrm{Aa}$ & $97,3 \mathrm{Ab}$ & $98,8 \mathrm{Aa}$ & $99,0 \mathrm{Aa}$ & $100,0 \mathrm{Aa}$ \\
\hline 2. Chuva $(15 \mathrm{~mm})$ - aplicação - chuva $(15 \mathrm{~mm}) 7$ DAA & $98,0 \mathrm{Ab}$ & $99,0 \mathrm{Aab}$ & $99,0 \mathrm{Aab}$ & $99,3 \mathrm{Aa}$ & $100,0 \mathrm{Aa}$ \\
\hline 3. Chuva $(15 \mathrm{~mm})$ - aplicação - chuva $(15 \mathrm{~mm}) 14$ DAA & $98,5 \mathrm{Ab}$ & $98,8 \mathrm{Aab}$ & $98,5 \mathrm{Ab}$ & $99,0 \mathrm{Aa}$ & $100,0 \mathrm{Aa}$ \\
\hline 4. Aplicação solo seco - chuva (15 mm) 14 DAA & $98,5 \mathrm{Ab}$ & $99,3 \mathrm{Aab}$ & $98,8 \mathrm{Aab}$ & 99,8 Aab & $100,0 \mathrm{Aa}$ \\
\hline $\mathrm{F}$ (profundidade) & \multicolumn{5}{|c|}{$6,59 * *$} \\
\hline $\mathrm{F}$ (umidade) & \multicolumn{5}{|c|}{$0,47^{\mathrm{ns}}$} \\
\hline $\mathrm{F}$ (prof. $\mathrm{x}$ umidade) & \multicolumn{5}{|c|}{$1,68^{*}$} \\
\hline CV $(\%)$ & \multicolumn{5}{|c|}{0,93} \\
\hline DMS & \multicolumn{5}{|c|}{1,30} \\
\hline
\end{tabular}

Médias seguidas de mesma letra, maiúscula na coluna e minúscula na linha, não diferem estatisticamente entre si pelo teste $\mathrm{t}(\mathrm{P}<0,05)$

${ }^{*}$ significativo a $5 \%$ de probabilidade; ${ }^{*}$ significativo a $1 \%$ de probabilidade; ${ }^{\text {ns }}$ não significativo.

Tabela 5 - Controle das plantas de Digitaria spp. nas diferentes profundidades de germinação pelo herbicida tebuthiuron, aos 28 DAA. Botucatu-SP - 2006/07

\begin{tabular}{|c|c|c|c|c|c|}
\hline \multirow{2}{*}{ Tratamento } & \multicolumn{5}{|c|}{ Profundidade $(\mathrm{cm})$} \\
\hline & 1 & 2 & 3 & 4 & 5 \\
\hline 1. Chuva de $15 \mathrm{~mm}$ e aplicação & $98,0 \mathrm{Ab}$ & $91,0 \mathrm{Bb}$ & $100,0 \mathrm{Aa}$ & $100,0 \mathrm{Aa}$ & $100,0 \mathrm{Aa}$ \\
\hline 2. Chuva (15 mm) - aplicação - chuva (15 mm) 7 DAA & $99,3 \mathrm{Aa}$ & $99,5 \mathrm{Aa}$ & $100,0 \mathrm{Aa}$ & $100,0 \mathrm{Aa}$ & $100,0 \mathrm{Aa}$ \\
\hline 3. Chuva $(15 \mathrm{~mm})$ - aplicação - chuva $(15 \mathrm{~mm}) 14 \mathrm{DAA}$ & $99,8 \mathrm{Aa}$ & $90,0 \mathrm{Bb}$ & $95,0 \mathrm{Aab}$ & $100,0 \mathrm{Aa}$ & $100,0 \mathrm{Aa}$ \\
\hline 4. Aplicação solo seco - chuva $(15 \mathrm{~mm}) 14 \mathrm{DAA}$ & $99,0 \mathrm{Aa}$ & $100,0 \mathrm{Aa}$ & $98,8 \mathrm{Aa}$ & $100,0 \mathrm{Aa}$ & $100,0 \mathrm{Aa}$ \\
\hline F (profundidade) & \multicolumn{5}{|c|}{$2,82 *$} \\
\hline F (umidade) & \multicolumn{5}{|c|}{$1,62^{\mathrm{ns}}$} \\
\hline $\mathrm{F}$ (prof. $\mathrm{x}$ umidade) & \multicolumn{5}{|c|}{$1,12^{\text {ns }}$} \\
\hline $\mathrm{CV}(\%)$ & \multicolumn{5}{|c|}{4,86} \\
\hline DMS & \multicolumn{5}{|c|}{6,77} \\
\hline
\end{tabular}

Médias seguidas de mesma letra, maiúscula na coluna e minúscula na linha, não diferem estatisticamente entre si pelo teste $\mathrm{t}(\mathrm{P}<0,05)$

${ }^{*}$ significativo a $5 \%$ de probabilidade; $* *$ significativo a $1 \%$ de probabilidade; ${ }^{\text {ns }}$ não significativo. 
Tabela 6 - Controle das plantas de Digitaria spp. nas diferentes profundidades de germinação pelo herbicida tebuthiuron, aos 42 DAA. Botucatu-SP - 2006/07

\begin{tabular}{|c|c|c|c|c|c|}
\hline \multirow{2}{*}{ Tratamento } & \multicolumn{5}{|c|}{ Profundidade $(\mathrm{cm})$} \\
\hline & 1 & 2 & 3 & 4 & 5 \\
\hline 1. Chuva de $15 \mathrm{~mm}$ e aplicação & $100,0 \mathrm{Aa}$ & $100,0 \mathrm{Aa}$ & $100,0 \mathrm{Aa}$ & $100,0 \mathrm{Aa}$ & $100,0 \mathrm{Aa}$ \\
\hline 2. Chuva (15 mm) - aplicação - chuva (15 mm) 7 DAA & $100,0 \mathrm{Aa}$ & $100,0 \mathrm{Aa}$ & $100,0 \mathrm{Aa}$ & $100,0 \mathrm{Aa}$ & $100,0 \mathrm{Aa}$ \\
\hline 3. Chuva $(15 \mathrm{~mm})$ - aplicação - chuva $(15 \mathrm{~mm}) 14$ DAA & $100,0 \mathrm{Aa}$ & $88,5 \mathrm{Bb}$ & $92,5 \mathrm{Aab}$ & $100,0 \mathrm{Aa}$ & $100,0 \mathrm{Aa}$ \\
\hline 4. Aplicação solo seco - chuva $(15 \mathrm{~mm}) 14 \mathrm{DAA}$ & $100,0 \mathrm{Aa}$ & $100,0 \mathrm{Aa}$ & $100,0 \mathrm{Aa}$ & $100,0 \mathrm{Aa}$ & $100,0 \mathrm{Aa}$ \\
\hline F (profundidade) & \multicolumn{5}{|c|}{$2,15^{*}$} \\
\hline F (umidade) & \multicolumn{5}{|c|}{$2,00^{\mathrm{ns}}$} \\
\hline $\mathrm{F}$ (prof. $\mathrm{x}$ umidade) & \multicolumn{5}{|c|}{$0,95^{\mathrm{ns}}$} \\
\hline CV $(\%)$ & \multicolumn{5}{|c|}{5,75} \\
\hline DMS & \multicolumn{5}{|c|}{8,01} \\
\hline
\end{tabular}

Médias seguidas de mesma letra, maiúscula na coluna e minúscula na linha, não diferem estatisticamente entre si pelo teste $\mathrm{t}(\mathrm{P}<0,05)$ ${ }^{*}$ significativo a $5 \%$ de probabilidade; ${ }^{*}$ significativo a $1 \%$ de probabilidade; ${ }^{\text {ns }}$ não significativo.

Tabela 7 - Massa seca (g) das plantas de Brachiaria plantaginea submetidas à aplicação do herbicida tebuthiuron, aos 42 DAA. Botucatu-SP - 2006/07

\begin{tabular}{|c|c|c|c|c|c|}
\hline \multirow{2}{*}{ Tratamento } & \multicolumn{5}{|c|}{ Profundidade $(\mathrm{cm})$} \\
\hline & 1 & 2 & 3 & 4 & 5 \\
\hline 1. Chuva de $15 \mathrm{~mm}$ e aplicação & $1,0 \mathrm{Bab}$ & $0,5 \mathrm{Bab}$ & $0,3 \mathrm{Bb}$ & $0,5 \mathrm{Bab}$ & $1,5 \mathrm{Ba}$ \\
\hline 2. Chuva $(15 \mathrm{~mm})$ - aplicação - chuva $(15 \mathrm{~mm}) 7$ DAA & $0,0 \mathrm{Ba}$ & $0,0 \mathrm{Ba}$ & $0,0 \mathrm{Ba}$ & $0,0 \mathrm{Ca}$ & $0,3 \mathrm{Ba}$ \\
\hline 3. Chuva $(15 \mathrm{~mm})$ - aplicação - chuva $(15 \mathrm{~mm}) 14$ DAA & $0,0 \mathrm{Ba}$ & $0,0 \mathrm{Ba}$ & $0,0 \mathrm{Ba}$ & $0,0 \mathrm{Ca}$ & $0,3 \mathrm{Ba}$ \\
\hline 4. Aplicação solo seco - chuva $(15 \mathrm{~mm}) 14 \mathrm{DAA}$ & $0,0 \mathrm{Ba}$ & $0,0 \mathrm{Ba}$ & $0,0 \mathrm{Ba}$ & $0,3 \mathrm{Ca}$ & $0,3 \mathrm{Ba}$ \\
\hline Testemunha sem aplicação & $9,3 \mathrm{Ac}$ & $9,5 \mathrm{Ab}$ & $9,0 \mathrm{Ac}$ & $12,3 \mathrm{Aa}$ & $10,8 \mathrm{Aa}$ \\
\hline F (profundidade) & \multicolumn{5}{|c|}{$5,46^{* *}$} \\
\hline F (umidade) & \multicolumn{5}{|c|}{$740,23^{* *}$} \\
\hline $\mathrm{F}$ (prof. $\mathrm{x}$ umidade) & \multicolumn{5}{|c|}{$2,61 * *$} \\
\hline CV $(\%)$ & \multicolumn{5}{|c|}{32,90} \\
\hline DMS & \multicolumn{5}{|c|}{1,03} \\
\hline
\end{tabular}

Médias seguidas de mesma letra, maiúscula na coluna e minúscula na linha, não diferem estatisticamente entre si pelo teste $\mathrm{t}(\mathrm{P}<0,05)$.

${ }^{*}$ significativo a $5 \%$ de probabilidade; $* *$ significativo a $1 \%$ de probabilidade; ${ }^{\text {ns }}$ não significativo.

Tabela 8 - Massa seca (g) das plantas de Digitaria spp. submetidas à aplicação do herbicida tebuthiuron, aos 42 DAA. Botucatu-SP $-2006 / 07$

\begin{tabular}{|c|c|c|c|c|c|}
\hline \multirow{2}{*}{ Tratamento } & \multicolumn{5}{|c|}{ Profundidade $(\mathrm{cm})$} \\
\hline & 1 & 2 & 3 & 4 & 5 \\
\hline 1. Chuva de $15 \mathrm{~mm}$ e aplicação & $0,00 \mathrm{Ba}$ & $0,00 \mathrm{Ba}$ & $0,00 \mathrm{Ba}$ & $0,00 \mathrm{Aa}$ & $0,00 \mathrm{Aa}$ \\
\hline 2. Chuva (15 mm) - aplicação - chuva (15 mm) 7 DAA & $0,00 \mathrm{Ba}$ & $0,00 \mathrm{Ba}$ & $0,00 \mathrm{Ba}$ & $0,00 \mathrm{Aa}$ & $0,00 \mathrm{Aa}$ \\
\hline 3. Chuva (15 mm) - aplicação - chuva $(15 \mathrm{~mm}) 14$ DAA & $0,00 \mathrm{Ba}$ & $0,00 \mathrm{Ba}$ & $0,00 \mathrm{Ba}$ & $0,00 \mathrm{Aa}$ & $0,00 \mathrm{Aa}$ \\
\hline 4. Aplicação solo seco - chuva (15 mm) 14 DAA & $0,00 \mathrm{Ba}$ & $0,00 \mathrm{Ba}$ & $0,00 \mathrm{Ba}$ & $0,00 \mathrm{Aa}$ & $0,00 \mathrm{Aa}$ \\
\hline Testemunha sem aplicação & $1,81 \mathrm{Aa}$ & $0,50 \mathrm{Ab}$ & $0,50 \mathrm{Ab}$ & $0,00 \mathrm{Ac}$ & $0,00 \mathrm{Ac}$ \\
\hline F (profundidade) & \multicolumn{5}{|c|}{$2,84^{*}$} \\
\hline F (umidade) & \multicolumn{5}{|c|}{$10,60 * *$} \\
\hline $\mathrm{F}$ (prof. $x$ umidade) & \multicolumn{5}{|c|}{$5,36^{* *}$} \\
\hline CV $(\%)$ & \multicolumn{5}{|c|}{77,28} \\
\hline DMS & \multicolumn{5}{|c|}{0,44} \\
\hline
\end{tabular}

Médias seguidas de mesma letra, maiúscula na coluna e minúscula na linha, não diferem estatisticamente entre si pelo teste $\mathrm{t}(\mathrm{P}<0,05)$

${ }^{*}$ significativo a $5 \%$ de probabilidade; ${ }^{*}$ significativo a $1 \%$ de probabilidade; ${ }^{\text {ns }}$ não significativo. 
Nas avaliações finais, ocorridas aos 42 DAA, demonstradas na Tabela 6, nota-se que, quando comparados os tratamentos, todos apresentaram excelente eficácia no controle dessa espécie de planta daninha nas diferentes profundidades de germinação testadas. Nesse caso, somente o tratamento com permanência do produto em solo úmido por 14 dias antes da precipitação, nas profundidades de 2 e $3 \mathrm{~cm}$, não apresentou controle total.

Os resultados de massa seca para Digitaria spp. (Tabela 8) demonstram acúmulo de biomassa até a profundidade de $3 \mathrm{~cm}$ na testemunha, bem como o excelente controle promovido pelo herbicida tebuthiuron nas condições estudadas.

\section{LITERATURA CITADA}

BALL, D. A. Weed seedbank response to tillage, herbicides, and crop rotation sequence. Weed Sci., v. 40, n.4 p. 654-659, 1992.

CANOSSA, R. S. et al. Profundidade de semeadura afetando a emergência de plântulas de Alternanthera tenella. Planta Daninha, v. 25, n. 4, p. 719-725, 2007.

CARMONA, R.; MURDOCH, A. J. Interactions of temperature and dormancy-relieving compounds on the germination of weed seeds. Seed Sci. Res., v. 5, n. 4, p. $227-236,1995$.

DIAS, N. M. P. et al. Identificação taxonômica de espécies de capim-colchão infestantes da cana-de-açúcar no Estado de São Paulo e eficácia de herbicidas no controle de Digitaria nuda. Bragantia, v. 64, n. 3, p. 389-396, 2005.

FERNÁNDEZ-QUINTANILLA, C. et al. Ecologia de lãs malas hierbas. In: GARCIA TORRES, L.; FERNÁNDEZQUINTANILLA, C. Fundamentos sobre malas hierbas y herbicidas. Madrid: Mundi-Prensa, 1991. p. 49-69.
FIRMINO, L. E. et al. Movimentação do herbicida imazapyr no perfil de solos tropicais. Planta Daninha, v. 26, n. 1, p. $223-230.2008$

GAZZIERO, D. L. P. et al. As plantas daninhas e a semeadura direta. Londrina: Embrapa Soja, 2001. 59 p.

JAVARONI, R. C. et al. Comportamento dos herbicidas atrazina e alaclor aplicados em solo preparado para o cultivo de cana-de-açúcar. Química Nova, v. 22 n. 1, p. 58-64, 1999.

NEGRISOLI, E. et al. Associação do herbicida tebuthiuron com a cobertura de palha no controle de plantas daninhas no sistema de cana-crua. Planta Daninha, v. 25, n. 3, p. $621-628,2007$

NEGRISOLI, E. et al. Eficácia do herbicida oxyfluorfen com a cobertura de palha no controle de plantas daninhas.

Planta Daninha, v. 27, n. 1, p. 197-2003, 2009.

ROBERTS, H. A. Seed banks in the soil. Cambridge: Academic Press, 1981. 55 p.

RODRIGUES, B. N.; ALMEIDA,F.S. Guia de herbicidas. Londrina: IAPAR, 2011. 697 p

SOCIEDADE BRASILEIRA DA CIÊNCIA DAS PLANTAS DANINHAS - SBCPD. Procedimentos para instalação, avaliação e análise de experimentos com herbicidas. Londrina: 1995. 42 p.

SCHWE1ZER, E. E.; ZIMDAHL, R. Li. Weed seed decline in irrigaled soil aftcr six years of continuous corn (Zea mays) and herbicides. Weed Sci., v. 32, p. 76-83, 1984.

SILVA, A. A. et al. Herbicidas: comportamento no solo. In: SILVA, A. A.; SILVA, J. F. Tópicos em manejo de plantas daninhas. Viçosa, MG: Universidade Federal de Viçosa, 2007. p. $189-248$.

ZANINE, A. M.; SANTOS, E. D. Competição entre espécies de plantas - uma revisão. R. Fac. Zootec. Vet. Agron., v. 11, n. 1, p. 103-122, 2004. 